\title{
La calidad del vino a la luz de la teoría de las convenciones: aplicación a las denominaciones de origen aragonesas
}

\section{Quality of wine in light of the convention theory: the Aragon designations of origin}

\author{
Enrique Ruiz Budría, Ana Castelló Puig, Eugenio Climent López, Ana \\ Isabel Escalona Orcao, Mariluz Hernández Navarro, Blanca Loscertales \\ Palomar* y Luisa María Frutos Mejías **
}

INTRODUCCIÓN: NUEVAS PERSPECTIVAS EN EL MERCADO INTERNACIONAL DEL VINO

Desde hace unas décadas, el mercado internacional del vino está sufriendo importantes modificaciones, tanto por la ampliación de la oferta, con la incorporación de nuevos productores, como por la diversificación de la demanda, marcada por un descenso en los países tradicionalmente consumidores y un incremento a escala mundial por la incorporación de otros nuevos.

Los datos existentes ponen de relieve el aumento de la producción mundial de vino a partir de los años noventa del pasado siglo, superándose actualmente los 300 millones de hectolitros. Los países europeos tradicionalmente productores se mantienen en cabeza y su oferta excede su propia demanda, por lo que deben buscar salida a sus excedentes en el mercado internacional, en competencia con los nuevos vitivinicultores «emergentes», especialmente

\footnotetext{
* Profesores Titulares de Universidad. Departamento de Geografía y Ordenación del Territorio. Grupo de Estudios de Desarrollo Territorial, Universidad de Zaragoza (GEDETUZ) (ecli ment@unizar.es).

${ }^{* * *}$ Catedrática de Universidad Emérita. Departamento de Geografía y Ordenación del Territorio. Grupo de Estudios de Desarrollo Territorial, Universidad de Zaragoza (GEDETUZ).
} 
California, Argentina, Australia, Sudáfrica y Chile, con zonas climáticas similares a las de la Europa de los viñedos.

La competencia por conseguir cuotas de mercado es, por tanto, muy fuerte, máxime cuando la importación se concentra en muy pocos países, si bien hay muchos otros en los que se está introduciendo el consumo de vino. A escala general, se observa un ascenso continuado de las exportaciones, con doce países líderes mundiales, ocupando España el tercer lugar, con una incidencia significativa en su balanza de pagos. Pero el ascenso de otros y el desajuste entre la producción y el consumo nacionales impulsaron al Gobierno español a plantear en 2004 una estrategia para el sector vitivinícola en el periodo 20072010, más allá de las normas impuestas por la Unión Europea (MAPA, 2004). En lo que concierne al comercio, el objetivo general se orientaba a convertir a España en líder del mercado mundial del vino, mejorando el valor que las ventas aportan al conjunto de la cadena de producción y distribución, capitalizando el crecimiento del consumo en países terceros, potenciando la promoción genérica y marquista y orientando la especialización de nuevos emprendedores vitivinícolas al mercado exterior, incluido el marketing. En pocas palabras: vender más y mejor.

Para ello se propusieron varios objetivos parciales encaminados a conseguir una conexión con los gustos de los consumidores, que se han decantado por la calidad pero con diversas características de vinos en distintos mercados y segmentos de los mismos. Para hacer un seguimiento del éxito de las estrategias propuestas se creó el Observatorio Español del Mercado del Vino, con participación pública y gestión privada, «como mecanismo fundamental del sector para el conocimiento exhaustivo de los mercados y las oportunidades que ofrecen, así como para la adaptación de los productos a ellos y el aprovechamiento de las oportunidades competitivas y fomentar la diferenciación de la oferta vinícola española para el mejor aprovechamiento de los distintos segmentos de mercado detectados y su adecuada promoción» (MAPA, 2004, p. 47). Las Denominaciones de Origen Protegidas (DOP) vitivinícolas no son ajenas a estos planteamientos, pero sus estrategias para conseguir expandir su mercado, aunque siempre busquen la calidad, pueden ser diversas.

En las páginas siguientes se tratará de profundizar en el conocimiento de dichas estrategias: en el apartado segundo se analiza el significado de las actuales transformaciones en los sistemas agroalimentarios y el concepto de calidad; en el apartado tercero se explica la teoría de las convenciones, como marco sistemático para la objetivación de los criterios de calidad y las diversas estrategias que los productores asumen ante un mercado que valora este concepto. De acuerdo con dicho marco teórico se presenta una batería de varia- 
bles estadísticas que sirven como indicadores objetivos de las convenciones vigentes en el mundo del vino y permiten la identificación de las estrategias de las DOP a la hora de ampliar sus cuotas de mercado y su presencia en los mercados internacionales por la vía de la mejora de la calidad. Dicha batería, que se expone en el apartado cuarto, ha sido puesta a prueba en un estudio de caso sobre las denominaciones de origen vitivinícolas de Aragón, cuyos resultados se recogen en el apartado quinto. En las conclusiones se discute su valor como herramienta metodológica para profundizar en el análisis de las estrategias que se guían por la lógica de la calidad en relación con el mercado ${ }^{1}$.

\section{EL SISTEMA AGROALIMENTARIO: ENTRE LA ESTANDARIZACIÓN Y LA DIFERENCIACIÓN}

En las últimas décadas el sistema agroalimentario se halla sometido a la tensión de dos tendencias contrapuestas: la estandarización y la diferenciación (Murdoch y Miele, 1999; Ilbery et al., 2005). La estandarización consiste en ajustar los productos y los procesos de producción a tipos, modelos o normas; lo que determina que el precio sea el mecanismo regulador de la competencia en el mercado. La diferenciación consiste en elaborar productos de características singulares, para lo cual normalmente es necesario utilizar procesos de producción especiales. En este caso, la competencia en el mercado no se rige por el precio: cuando los consumidores eligen un producto diferenciado lo hacen porque encuentran en él ciertas propiedades que satisfacen sus necesidades o sus gustos y que no tienen otros. El conjunto de las propiedades que hacen diferentes a los productos y que constituyen la base de su éxito en los mercados suele etiquetarse con una palabra genérica: calidad. Así, la tensión señalada al principio se completa de esta manera: estandarización, con competencia en precios, frente a diferenciación, con competencia en calidad.

Los procesos de estandarización en el sistema agroalimentario que vienen produciéndose desde hace décadas, afectan a los métodos de producción y a los hábitos de consumo de la población, que se van haciendo cada vez más uniformes. El protagonismo de las grandes empresas productoras multinacionales en dicho proceso es patente, ya que introducen y difunden pautas estandarizadas

1 El trabajo presenta resultados del proyecto de investigación CSO2008-05793-C0302/GEOG ("Gobernanza, innovación y convenciones en las comarcas vitivinícolas de Aragón: tipología y prospectiva de denominaciones de origen a partir de la teoría de los mundos de producción"), financiado por el Ministerio de Ciencia e Innovación y los Fondos Europeos de Desarrollo Regional (FEDER). 
de producción en serie a la vez que promocionan marcas comerciales registradas y reconocidas universalmente. También participan en ese proceso las grandes superficies de venta al por menor, fomentando hábitos de consumo uniformes.

Pero junto a esta tendencia se observa igualmente la emergencia de empresas, tanto individuales como agrupadas en organizaciones de diversa naturaleza, que propugnan un sistema agroalimentario basado en productos y prácticas de producción no estandarizadas (Ilbery, 2001; Ilbery et al., 2005), que se orientan a un nuevo modelo de consumidor y buscan dar respuesta a algunos graves problemas sanitarios asociados a determinados procesos industriales. Respecto a lo primero, en los países avanzados son cada vez más los consumidores que no se conforman con quedar subsumidos en una masa de gustos uniformes y tratan de diferenciarse mediante pautas de consumo personalizadas que, en todo caso, implican la demanda de mayor variedad de productos. Respecto a la segunda, una serie de crisis alimentarias, como la llamada «enfermedad de las vacas locas», la gripe aviar o más recientemente la «crisis de la bacteria Escherichia coli o del pepino», junto con las noticias sobre los daños a la salud que pueden provocar los pesticidas, los aditivos y otros productos de uso frecuente en la agricultura, la ganadería y la industria de alimentación, han extendido entre los consumidores una cierta desconfianza frente a los procesos de producción estandarizados del complejo agroalimentario, tanto en el segmento agrario como en el manufacturero. La demanda de alimentos más saludables y seguros orienta las pautas de consumo hacia empresas con fuerte arraigo local que utilizan métodos de producción artesanales. Ambos rasgos dan mayor confianza al consumidor, porque el carácter local le permite una identificación más precisa de la empresa y del producto y porque los alimentos artesanos le parecen más naturales y menos manipulados que los industriales. En otras palabras, es «la diferenciación entre el paradigma agroindustrial y los «alimentos alternativos» o lo que recibe el nombre de «giro hacia la calidad» dentro del sector agroalimentario. Un elemento de importancia en este giro es la perspectiva del espacio o, mejor, de lo local» (Straete, 2004, p. 228).

El crecimiento del número de consumidores que demandan alimentos diferenciados y saludables es tan notorio que las grandes multinacionales de la industria agroalimentaria tratan de dar respuesta a esas nuevas actitudes diversificando sus gamas de productos, y a menudo también, recurriendo al procedimiento de comprar empresas de fuerte arraigo local que se integran en el grupo conservando su nombre y demás señas de identidad. Por su parte, las grandes superficies de distribución exhiben en sus lineales una variada gama de alimentos, a la vez que crean secciones especiales abiertas a las empresas de productos locales alternativos, con las que frecuentemente se establecen 
acuerdos de comercialización que implican que el nombre del distribuidor aparezca junto al del fabricante, como forma de mostrar el compromiso de aquél con este tipo de productos. Al adoptar estas pautas, las grandes empresas del sistema agroalimentario, aunque sean las principales impulsoras de los procesos de estandarización, están reconociendo implícitamente que ésta no es la única alternativa y buscan activamente participar también en el negocio de los procesos de producción de carácter artesanal y local. En resumen, «podemos afirmar que el sector de la alimentación se está bifurcando en dos «zonas» principales de producción: por una parte, las redes de alimentos globales, estandarizados e industrializados; por otra los procesos de producción localizados y especializados» (Murdoch y Miele, 1999, p. 469).

Ambas formas de producción se rigen por lógicas productivas y comerciales diferentes: la de los alimentos estandarizados, dada la homogeneidad de los productos, se guía por la lógica del precio, de manera que las estrategias de fabricantes y distribuidores se orientan preferentemente a reducir los costes para lanzar al mercado productos más baratos que sus competidores. La producción localizada y especializada se rige por la lógica de la diferenciación, estrechamente ligada a ese nuevo paradigma de la calidad, asumiéndose que los consumidores están dispuestos a pagar más por unos productos acordes a sus gustos personales y sus exigencias de salud.

En la mayor parte de los sectores agroalimentarios unas empresas trabajan en el ámbito de los alimentos estandarizados y otras en la de los alimentos especializados e incluso algunas lo hacen en ambas. ¿Cuál es la situación del sector del vino? «La que durante siglos fue considerada una industria de campesinos se está caracterizando por la presencia de grandes empresas multinacionales. Al mismo tiempo, el nivel de concentración empresarial de la producción en esta industria está muy por debajo del de otras del sector de la alimentación, como el café, el cacao o la cerveza. El comercio al por menor de vino, que tradicionalmente ha sido el dominio de pequeñas tiendas especializadas, está ahora en manos de las cadenas de supermercados [...] Aunque existe el temor a la homogeneización de estilos y ofertas en el mercado del vino, es una industria que todavía elabora una enorme variedad de productos diferentes, que se venden bajo una combinación de marcas comerciales, variedades de uva e indicaciones geográficas de origen» (Ponte, 2009, p. 238). El texto refleja que en el sector del vino se da también esa bifurcación en dos «zonas»: la creciente presencia de empresas multinacionales y grandes superficies de distribución podría tomarse como indicio de estandarización, pero el alto grado de diferenciación del producto y la importancia de las denominaciones de origen apuntan a la especialización. No obstante, teniendo en 
cuenta que las grandes empresas intentan cubrir ambos campos y dado que la producción vinícola presenta un grado de concentración muy inferior al de otros sectores agroalimentarios, podría aceptarse como punto de partida que el sector del vino se desenvuelve preferentemente en la «zona» de alimentos localizados y especializados, lo cual significa que se regiría más bien por la lógica de la diferenciación y la calidad que por la lógica del precio.

El precio es una variable cuantitativa cuyos mecanismos de formación se conocen bastante bien, pero ¿qué es la calidad? Dada la carga subjetiva de este concepto y sus posibles variaciones en el tiempo, la respuesta no es fácil y no son pocos los esfuerzos que se han hecho para introducir el concepto de forma operativa en el análisis científico, sin que pueda decirse que la tarea esté acabada. Una de las líneas de trabajo más interesantes en esta dirección es la teoría de las convenciones, a cuya exposición va dedicado el apartado siguiente.

\section{El MARCO TEÓRICO: LA CALIDAD DESDE LA TEORÍA DE LAS CONVENCIONES}

La teoría de las convenciones surge como resultado de centrar la atención en las cuestiones que plantea el acto mismo de calificar o juzgar, tanto en la investigación científica como en la acción social. Boltanski y Thévenot (1991) identifican en dicho acto una pluralidad de formas de generalización, que a su vez constituyen formas de justificación de la acción. Ejemplificando esto en el ámbito de la vitivinicultura, valorar la calidad de un vino (lo mismo si lo hace el consumidor, que el productor o el experto) es un acto de calificar, un juicio, que se hace a partir de una idea general sobre lo que es la calidad, idea que sirve también como base para justificar o censurar la estrategia de producción y comercialización que ha conducido a la elaboración y distribución de dicho vino. Pero diferentes evaluadores pueden utilizar distintas formas de generalización, lo que significa que la valoración de la calidad del vino puede ser muy diferente en función de la que se utilice. Boltanski y Thévenot (1991) distinguen seis formas de generalización y, por tanto, de justificación: la de la inspiración, la industrial, la mercantil, la doméstica, la de la opinión y la cívica.

Cada una de ellas da lugar a una categoría diferente de convenciones, que se definen como «prácticas, rutinas, acuerdos y sus estructuras asociadas, informales o institucionales, que aúnan las acciones por las mutuas expectativas» (Salais and Storper, 1992, p. 171). En el ámbito de la actividad industrial esta teoría aporta criterios para la valoración de la calidad de los productos y de los procesos de producción que determinan dicha calidad, así como de las demandas de los consumidores, que son las señales que reciben las empresas para ajustar su oferta (Escalona et al., 2010 y 2011). 
A la categoría de la inspiración no se le otorga apenas relevancia en el ámbito de la actividad económica; de hecho, Murdoch y Miele, cuyos trabajos tienen mucha influencia en el estudio de los sistemas agroalimentarios, no la toman en consideración. Por el contrario, es una categoría fundamental en el mundo del arte, donde la calificación se realiza tomando como criterio central la creatividad. Tendría sentido su aplicación en la vitivinicultura, por ejemplo, a la hora de calificar determinados vinos de autor, que buscan sorprender a los consumidores con novedades muy creativas, pero lo cierto es que apenas se hace.

En la categoría de las convenciones industriales la calidad de los productos se evalúa sobre la base de su eficiencia y fiabilidad, para lo cual se establecen parámetros objetivos y mensurables desde el punto de vista técnico. La productividad es el criterio clave para las empresas. En el sector vitivinícola tiene que ver fundamentalmente con los procesos de producción encaminados a obtener una alta productividad.

En la categoría de las convenciones mercantiles o comerciales la calidad de los productos se evalúa en relación con los principios que rigen el mercado, siendo los criterios básicos el precio y la utilidad. El criterio clave para las empresas es la competitividad. En el apartado anterior se ha planteado una contraposición entre calidad y precio, que daría lugar a dos lógicas distintas, cada una de las cuales rige una de las dos «zonas» de producción de que hablan Murdoch y Miele (1999), pero la teoría de las convenciones, como se ve, rompe esa dicotomía considerando que el precio no es algo contrapuesto a la calidad, sino uno de los criterios que pueden utilizarse para valorarla.

En la categoría de las convenciones domésticas la calidad de los productos se evalúa en términos de vinculación a un lugar y una tradición determinados. El criterio clave para las empresas es la confianza. Los consumidores valoran que un producto hecho en un lugar concreto (por empresas arraigadas en él y siguiendo los métodos de producción de siempre), tienen una calidad acreditada. Los productores tienen que ganarse la confianza de los consumidores asumiendo un compromiso firme en cuanto al origen local de las materias primas y el carácter tradicional de los métodos. Ese compromiso puede realizarse en forma colectiva, dando lugar a mecanismos de acreditación del tipo de las Denominaciones de Origen e Indicaciones Geográficas.

La categoría de las convenciones domésticas está en la base de la «zona» de procesos de producción localizados y especializados a que se refieren Murdoch y Miele (1999). Pero ello no debe inducir a confusión, suscitando una falsa contraposición entre estas convenciones y las industriales y las comerciales: no se puede perder de vista que se trata de categorías diferentes y que en esa «zona» también se toman en consideración la productividad, el precio y la 
demanda de los consumidores, puesto que su objetivo final es vender en el mercado nacional e internacional.

La categoría de convenciones de la opinión, también denominadas convenciones públicas, hacen referencia a la fama de los productos, es decir, al hecho de que sus marcas, logotipos, etiquetas o presentación comercial, sean fácilmente reconocidos por los consumidores. El criterio clave para las empresas es la reputación. En esta categoría juegan un papel muy importante los creadores de opinión, es decir, quienes tienen acreditada una reputación como expertos.

Las convenciones públicas juegan un papel muy destacado en la vitivinicultura, especialmente de cara a su inserción en el mercado internacional, donde existen Guías de Vinos que gozan de gran prestigio y concursos a los que concurren muchas empresas, que hacen publicidad de los premios obtenidos, así como consumidores muy atentos a las noticias del sector y a las opiniones de los expertos. Cabe añadir que el reconocimiento de las marcas no se refiere sólo a las empresas individuales, sino también a las Denominaciones de Origen e Indicaciones Geográficas, que suelen presentarse en el mercado como marcas colectivas, cuya reputación afecta a todas las bodegas que forman parte de ellas.

En la categoría de las convenciones cívicas la calidad de los productos es evaluada en términos de los beneficios sociales generales, es decir, de su aportación al bienestar de los ciudadanos. El criterio clave para las empresas es responsabilidad. Este grupo de convenciones hace referencia, por una parte, a aspectos como la salud y la seguridad de los consumidores, incluyéndose, entre otras, todo el conjunto de normas e instituciones relativas a los registros sanitarios de los alimentos, a los requisitos que deben reunir los envases y a la información que deben proporcionar las etiquetas respecto a los ingredientes. Por otra parte, incluyen también determinadas normas de carácter ético, como las que condenan el trabajo infantil o las que promueven el comercio justo, así como las que se refieren a la calidad de las condiciones de trabajo o a la devolución a la sociedad de parte de los beneficios vía patronazgo u otras.

En la categoría de las cívicas, tal como proponen Boltanski y Thévenot (1991), se incluyen también las normas que hacen referencia al medio ambiente, pero posteriormente Murdoch et al. (2000) propusieron crear con ellas una nueva categoría, a la que denominaron ecológica. La calidad en este ámbito se valora en términos de si los procesos y productos son dañinos desde el punto de vista medioambiental o si, por el contrario, se ajustan a criterios de sostenibilidad, que sería la palabra clave para las empresas.

Recapitulando brevemente, la teoría de las convenciones ofrece un marco teórico general para abordar el problema sobre qué es la calidad y cómo eva- 
luarla. Muestra que efectivamente no es una tarea fácil, puesto que la calidad es algo convencional y multidimensional.

METODOLOGÍA Y FUENTES: VARIABLES PARA IDENTIFICAR Y EVALUAR LAS CONVENCIONES

La teoría de las convenciones ha servido de marco general a diversas investigaciones sobre el sector agroalimentario. Pueden citarse, a modo de ejemplo, los estudios comparados sobre dos tipos de queso en Francia y Noruega (Amilien et al., 2007) o sobre la salazón de pescado en Noruega y el vino en la región española de Castilla y León (Lindkvist y Sánchez, 2008); asimismo, los estudios monográficos sobre una empresa de yogur en Gales (Murdoch et al., 2000), sobre las granjas de leche en Noruega (Straete, 2004) o sobre el vino en África del Sur (Ponte, 2009) y en Castilla y León (Sánchez et al., 2010). Todos ellos realizan una rigurosa exposición de la teoría, en términos generales o enfatizando algún aspecto concreto de la misma, que luego aplican o ilustran mediante casos de estudio. En éstos, en general, se utiliza una metodología basada en entrevistas y otras técnicas cualitativas. El caso se interpreta desde la teoría, a partir de la información obtenida, dando lugar a aportaciones muy sugerentes sobre las estrategias de las empresas o sobre la percepción de los consumidores respecto a la calidad de los productos.

Un problema de esta metodología es que las respuestas de los entrevistados pueden estar condicionadas por la manera de enunciar las preguntas o por la propia actitud del entrevistador. Además, es el investigador quien prepara los guiones o cuestionarios y a la vez quien interpreta las respuestas, lo cual hace que el proceso de investigación sea muy sensible a la subjetividad. Con esto no se pretende cuestionar la validez de la investigación cualitativa, que dispone de recursos metodológicos suficientes para minimizar dichos problemas, sino plantear la conveniencia de contrastarla o complementarla, siempre que sea posible, por medio de análisis cuantitativos. El propósito de este trabajo es seleccionar datos estadísticos objetivos, por cuanto proceden de fuentes de información independientes del investigador y del propio proceso de investigación, y someterlos a un análisis riguroso que neutralice la posible subjetividad de la interpretación.

En concreto, se trata de definir, para cada una de las categorías de convenciones descritas en el apartado anterior, una serie de variables cuantitativas que puedan calcularse a partir de datos publicados o fácilmente accesibles y que, según los valores que adopten, indiquen la importancia que tiene cada una de dichas categorías en el caso de estudio. Las variables definidas se reco- 
gen en el cuadro 4; se trata en total de 25 , correspondiendo 5 a cada una de las categorías de convenciones industriales, comerciales, domésticas, públicas y cívicas. No se ha tomado en consideración la categoría de la inspiración o creatividad, que no suele ser tenida en cuenta en los estudios sobre la realidad económica, según se ha indicado en el apartado anterior. Tampoco se han considerado como categoría aparte las convenciones ecológicas, que se han incluido en el bloque de las cívicas.

Una parte de las variables elegidas como indicadores de la importancia de las convenciones industriales tiene que ver directamente con la productividad, puesto que es el concepto clave de esta categoría: los porcentajes de superficie plantada con variedades de uva de alto rendimiento y con viñas cultivadas en espaldera. Otras indican la aplicación de economías de escala en el proceso industrial de transformación del vino: producción media de vino, como indicador del tamaño de las empresas, o porcentaje de vino joven, como indicador de la simplificación de los procesos. Estas variables son adecuadas para identificar una estrategia de penetración en los mercados internacionales orientada al segmento de mercado de masas, en el que los consumidores requieren vinos estandarizados con una buena relación calidad-precio.

Para identificar y valorar la importancia de las convenciones comerciales o de mercado se han seleccionado variables que ponen en evidencia la voluntad de satisfacer las demandas de los consumidores, como los segmentos de mercado a los que se dirigen los diferentes vinos de las bodegas y los países a los que se exportan. Se ha elegido también el cultivo de las variedades de uva llamadas globales, que fueron a partir de los años ochenta del pasado siglo las más apreciadas en el mercado internacional; su difusión se considera un buen indicador de la importancia que los viticultores dan a la rentabilidad, que es una cuestión clave en esta categoría. Por otra parte, se ha considerado que la atracción de bodegas foráneas es un buen indicador de las perspectivas de rentabilidad que ofrece un territorio.

Respecto a las convenciones domésticas se han seleccionado variables que indican el arraigo en un territorio determinado: la importancia de las variedades autóctonas de uva, de las bodegas autóctonas y de las marcas que hacen referencia a lo local. Otra de las variables remite a las formas tradicionales de relación entre los productores y los consumidores, como la venta de vino en la propia bodega, cuyo impacto en el comercio se produce indirectamente por medio del turismo: los turistas que compran en la propia bodega luego demandan esos vinos desde sus lugares de residencia.

Las variables seleccionadas como indicadores de las convenciones públicas hacen referencia a datos que ponen de relieve la fama o reputación de las em- 
presas: premios obtenidos en concursos, estrellas otorgadas en guías especializadas, menciones recibidas en revistas del sector y resultados obtenidos a través de los buscadores de Internet. Los dos últimos evidencian la fama en el sentido de popularidad, mientras que en los primeros juegan un papel especialmente significativo los juicios de los expertos, incidiendo ambos en la opinión y en la toma de decisiones de los consumidores.

Para las convenciones cívicas, por una parte, se han seleccionado variables que evidencian la preocupación de las empresas por los métodos de producción respetuosos con el medio ambiente: bodegas con certificación de producto ecológico y proporción de vino certificado como ecológico. Por otra parte, se han buscado otras que indiquen el interés por distribuir ampliamente los beneficios económicos del sector vitivinícola entre la sociedad: la importancia de las cooperativas y, en el caso de las denominaciones de origen, el grado de participación en las mismas de las bodegas del territorio.

Las fuentes de información de las que obtener los datos con los que construir las variables son diversas: por una parte están las bases de datos de los organismos administrativos competentes (Ministerio de Agricultura, Alimentación y Medio Ambiente y las Consejerías de Agricultura de las Comunidades Autónomas) y las de instituciones público-privadas (Consejos Reguladores de las Denominaciones de Origen, Comités Regionales de Agricultura Ecológica y Cámaras de Comercio). Por otra parte están las publicaciones especializadas (Anuarios de Bodegas de El Mundo y El País, revistas electrónicas, Guías y otras publicaciones científicas). Finalmente, en Internet ofrecen información tanto los buscadores como las páginas Web de Denominaciones de Origen, las bodegas y las entidades organizadoras de certámenes y premios.

Las variables obtenidas son de naturaleza muy diferente: porcentajes, promedios, valores absolutos e índices de cierta complejidad. Para poder trabajar con todas estas de forma conjunta es necesario normalizarlas previamente, lo cual se ha llevado a cabo mediante el cálculo del número $\mathrm{Z}$, cociente cuyo numerador es la diferencia entre el valor a normalizar y la media aritmética de los valores de la variable y cuyo denominador es la desviación estándar; los números $\mathrm{Z}$ tienen la propiedad de que su media aritmética es igual a 0 y su desviación estándar a 1.

\section{Estudio de Caso: las Denominaciones de Origen Protegidas de Aragón}

La metodología descrita en el apartado anterior ha sido testada en un estudio de caso sobre las cuatro Denominaciones de Origen Protegidas (DOP) del sector del vino que hay en Aragón (figura 1). Enumeradas por orden de anti- 
güedad son las de Cariñena, Campo de Borja, Somontano y Calatayud. Cariñena pertenece a la primera generación española de DOP y fue reconocida en 1932; el reconocimiento de las otras tres es mucho más reciente y tuvo lugar en la década de los ochenta (1980, 1984 y 1989, respectivamente).

\section{Caracterización de las Denominaciones de Origen Protegidas de Aragón}

En Aragón, como en la mayor parte de España, la vitivinicultura es una actividad económica tradicional, que hunde sus raíces profundamente en la historia. En la actualidad la superficie ocupada por viñedo en los territorios de

FIGURA 1

LOCALIZACIÓN DE LAS DOP ARAGONESAS

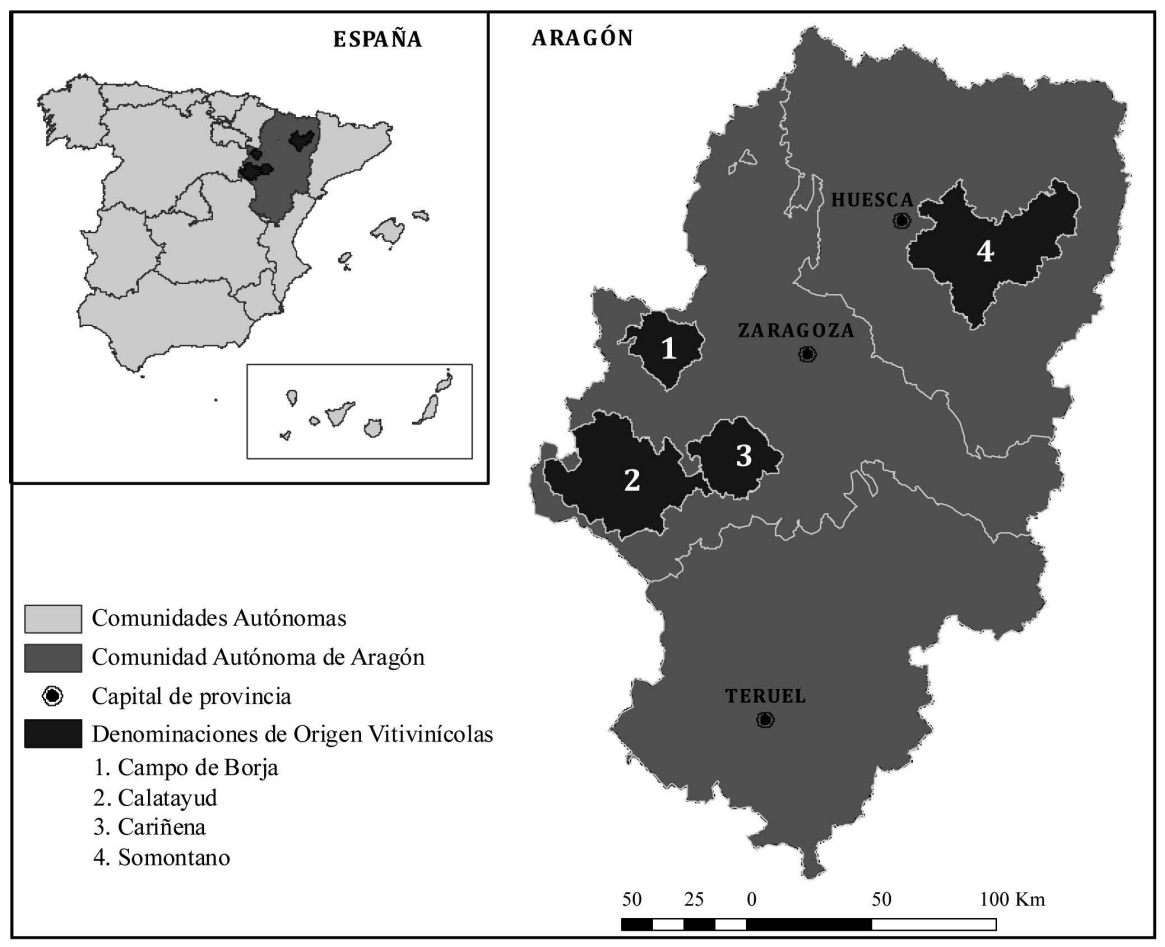

Fuente: elaboración propia a partir de la información procedente de la web oficial de la DOP Campo de Borja (http://www.campodeborja.com/geogra.htm), DOP Calatayud (http://www.docalatayud.com/), DOP Campo de Cariñena (http://www.docarinena.com/) y DOP Somontano (http://dosomontano.com/). 
las cuatro DOP supera las 31.000 hectáreas, ocupa a casi 5.000 viticultores y mantiene en producción a 122 bodegas, una parte significativa de las cuales son cooperativas, para una producción total de 727.000 hectolitros de vino (cuadro 1). Por tamaño, Cariñena es, con diferencia, la DOP más importante de Aragón, con porcentajes de participación que oscilan entre el 45 y el 55\% en los parámetros señalados. Con respecto a la ocupación del terrazgo, destaca la importante presencia de la garnacha, variedad local mayoritaria en las DOP de Calatayud (67,3\%), Campo de Borja $(59,3 \%)$ y, en menor medida, Cariñena (33\%). La DOP Somontano presenta unas características totalmente diferentes en la composición de su viñedo, ocupado en su mayor parte por variedades foráneas: Cabernet-Sauvignon $(29,1 \%)$ y Merlot $(23,4 \%)$ (Loscertales, 2009; Castelló et al., 2010; DGA, 2010).

Es importante destacar la vocación exportadora de las DOP aragonesas, ya que en conjunto casi el 50\% del vino producido se consume en el extranjero. La saturación de los mercados con vinos elaborados a partir de variedades «globales» (Cabernet, Merlot, Chardonnay) y la fuerte competencia han revitalizado las variedades tradicionales, generalmente menos productivas, pero mejor adaptadas al medio geográfico (DGA, 2010, p. 6). Esta situación favorece a los vinos elaborados con garnacha y de ahí la fuerte presencia de los

\section{CUAdro 1}

CARACTERIZACIÓN DE LAS DOP VITIVINÍCOLAS DE ARAGÓN (2009)

\begin{tabular}{|c|c|c|c|c|}
\hline DOP & Calatayud & Campo de Borja & Cariñena & Somontano \\
\hline Superficie inscrita (ha) & 3.966 & 7.413 & 15.059 & 4.704 \\
\hline \multirow{3}{*}{$\begin{array}{l}\text { Principales variedades } \\
\text { (porcentajes sobre la } \\
\text { superficie inscrita) }\end{array}$} & Garnacha $(67,3)$ & Garnacha $(59,3)$ & Garnacha $(33,0)$ & Cabernet $(29,1)$ \\
\hline & Tempranillo $(15,3)$ & Tempranillo $(13,5)$ & Tempranillo $(25,6)$ & Tempranillo $(14,5)$ \\
\hline & Macabeo $(7,7)$ & Syrah $(8,4)$ & Macabeo $(14,8)$ & Merlot $(23,4)$ \\
\hline Viticultores & 1.100 & 1.294 & 1.984 & 508 \\
\hline Bodegas totales & 16 & 17 & 55 & 34 \\
\hline $\begin{array}{l}\text { Producción de vino } \\
\text { calificado (hectolitros: } \\
\text { media 2007-2009) }\end{array}$ & 58.047 & 138.659 & 401.185 & 129.113 \\
\hline Porcentaje exportado & 53,5 & 67,5 & 65,0 & 25,6 \\
\hline
\end{tabular}


aragoneses en el mercado internacional, hasta el punto de que la DOP Campo de Borja, autodenominada «el imperio de la garnacha», exporta el $67 \%$ de su producción. En el extremo opuesto, la DOP Somontano, que apostó por las variedades foráneas, se orienta en mayor medida al mercado nacional, exportando sólo el $25 \%$ de lo que produce.

El destino mayoritario de estos vinos es la Unión Europea, donde se dirige el 70\% de las exportaciones (cuadro 2). La DOP Cariñena depende de los mercados de Alemania y del Reino Unido, donde coloca casi el 60\% de sus ventas al exterior, mientras que el Somontano, se orienta más hacia los Países Bajos. Las exportaciones de las DOP Calatayud y Campo de Borja se dirigen hacia el mercado americano, donde son apreciados los vinos con marchamo local; Estados Unidos y Canadá absorben el 58\% de las ventas exteriores de la primera y el $31 \%$ de la segunda.

Las cuatro DOP aragonesas aportaron, en la campaña 2008-2009, porcentajes significativos del total de las españolas: el 4,9 de la superficie de viñedo, el 3,3 de los viticultores, el 2,5 de las bodegas, el 5,5 de la producción y el 8 por ciento de la exportación. Considerado en su conjunto, puede afirmarse del sector vitivinícola aragonés lo siguiente: primero, que es de alto rendimiento y productividad, puesto que es mayor el porcentaje de producción que los de superficie y fuerza de trabajo; segundo, que el tamaño de las bodegas es superior a la media nacional, pues la proporción de bodegas es menor que la

\section{CuAdro 2}

PRINCIPALES DESTINOS DE LAS EXPORTACIONES DE LAS DOP ARAGONESAS (PORCENTAJES SOBRE EL TOTAL EXPORTADO POR CADA DOP)

\begin{tabular}{lcccc}
\hline \multicolumn{1}{c}{ DOP } & Calatayud & Campo de Borja & Cariñena & Somontano \\
\hline Alemania & 14,8 & & 35,8 & 12,4 \\
Bélgica & 5,6 & & & 21,6 \\
Holanda & 5,8 & 17,6 & 6,0 & 25,3 \\
Reino Unido & 4,2 & 32,4 & 22,6 & \\
Suiza & & & & 8,5 \\
Canadá & 1,5 & 11,1 & 5,3 & \\
Estados Unidos & 56,5 & 19,9 & 6,3 & \\
Unión Europea (total) & 32,7 & 58,5 & 77,3 & 75,4 \\
\hline
\end{tabular}

Fuente: elaboración propia a partir de Ministerio de Agricultura, Alimentación y Medio Ambiente (2010): "Datos de las Denominaciones de Vinos y Bebidas Espirituosas". Madrid, Dirección General de Alimentación, Diputación General de Aragón. 
de producción y, tercero, que manifiesta una clara vocación exportadora, pues la exportación es la variable que presenta el porcentaje más alto de todas. La DOP Cariñena, que es la más grande de las cuatro, ocupa puestos destacados entre las DOP españolas: sobre un total de 85, es la decimonovena respecto al número de bodegas, la onceava respecto a la superficie, la novena respecto a producción y la sexta respecto a la exportación.

Tanto por la posición destacada de la DOP de Cariñena como la significativa participación de las cuatro aragonesas en el conjunto nacional, especialmente en lo que respecta a la presencia en los mercados internacionales, avalan su elección como caso de estudio con el que poner a prueba una metodología diseñada para identificar y valorar objetivamente la calidad del vino.

\section{Las convenciones en las Denominaciones de Origen Protegidas de Aragón}

Siguiendo el procedimiento previamente descrito, se han obtenido los valores de las veinticinco variables indicadas para cada una de las DOP y se han normalizado por medio de los números Z (cuadro 4). La media aritmética de éstos en cada categoría de convenciones (índice Z) permite conocer las orientaciones preferentes de las DOP en cuanto a la calidad del vino. Los valores del índice $Z$ tienen un significado fundamentalmente ordinal: permiten comprobar la posición relativa de cada DOP respecto a las demás, pudiendo tomarse como medida aproximada de la importancia que se otorga a cada categoría de convenciones.

El cuadro 3 recoge dichos índices Z, con cuyos valores se han realizado los gráficos radiales correspondientes a cada DOP (figura 2). Las diferencias entre

\section{CuAdro 3}

ÍNDICES Z DE LAS DOP ARAGONESAS RESPECTO A LAS CATEGORIAS DE CONVENCIONES

\begin{tabular}{lccccc}
\hline DENOMINACIONES DE & \multicolumn{5}{c}{ CATEGORÍA DE CONVENCIONES } \\
\cline { 2 - 6 } ORIGEN PROTEGIDAS & Industrial & Comercial & Doméstica & Pública & Cívica \\
\hline Calatayud & $-0,44$ & $-0,68$ & 0,51 & $-0,45$ & 0,05 \\
Campo de Borja & 0,45 & 0,61 & $-0,23$ & 1,01 & 0,43 \\
Cariñena & 0,19 & $-0,19$ & $-0,55$ & $-0,81$ & $-0,06$ \\
Somontano & $-0,20$ & 0,26 & 0,27 & 0,24 & $-0,41$ \\
\hline
\end{tabular}

Fuente: elaboración propia a partir de promedios de los valores recogidos en el cuadro 4. 


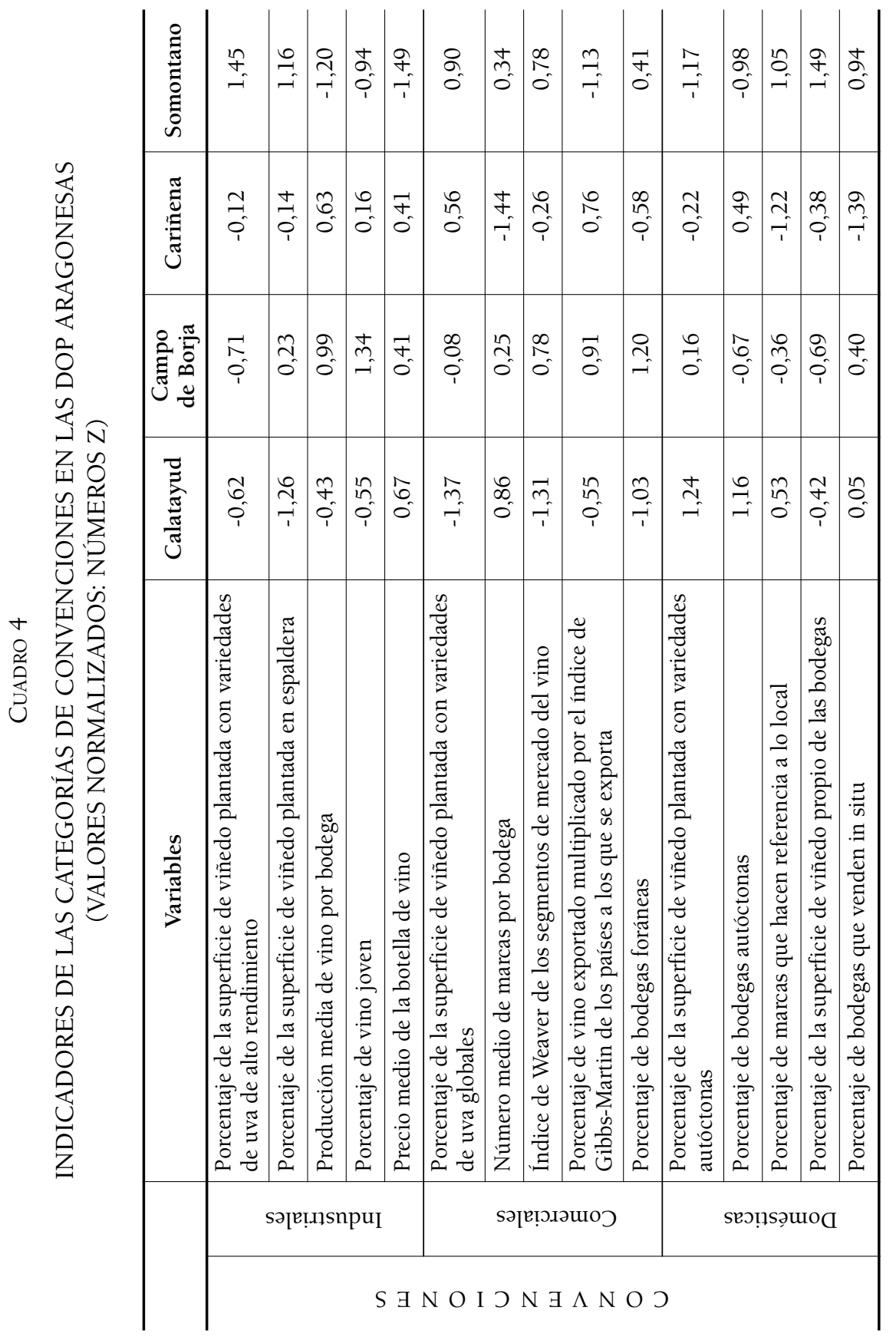

Estudios Geográficos, Vol. LXXIV, 274, pp. 231-254, enero-junio 2013

ISSN: 0014-1496, eISSN: 1988-8546, doi: 10.3989/estgeogr.201308 


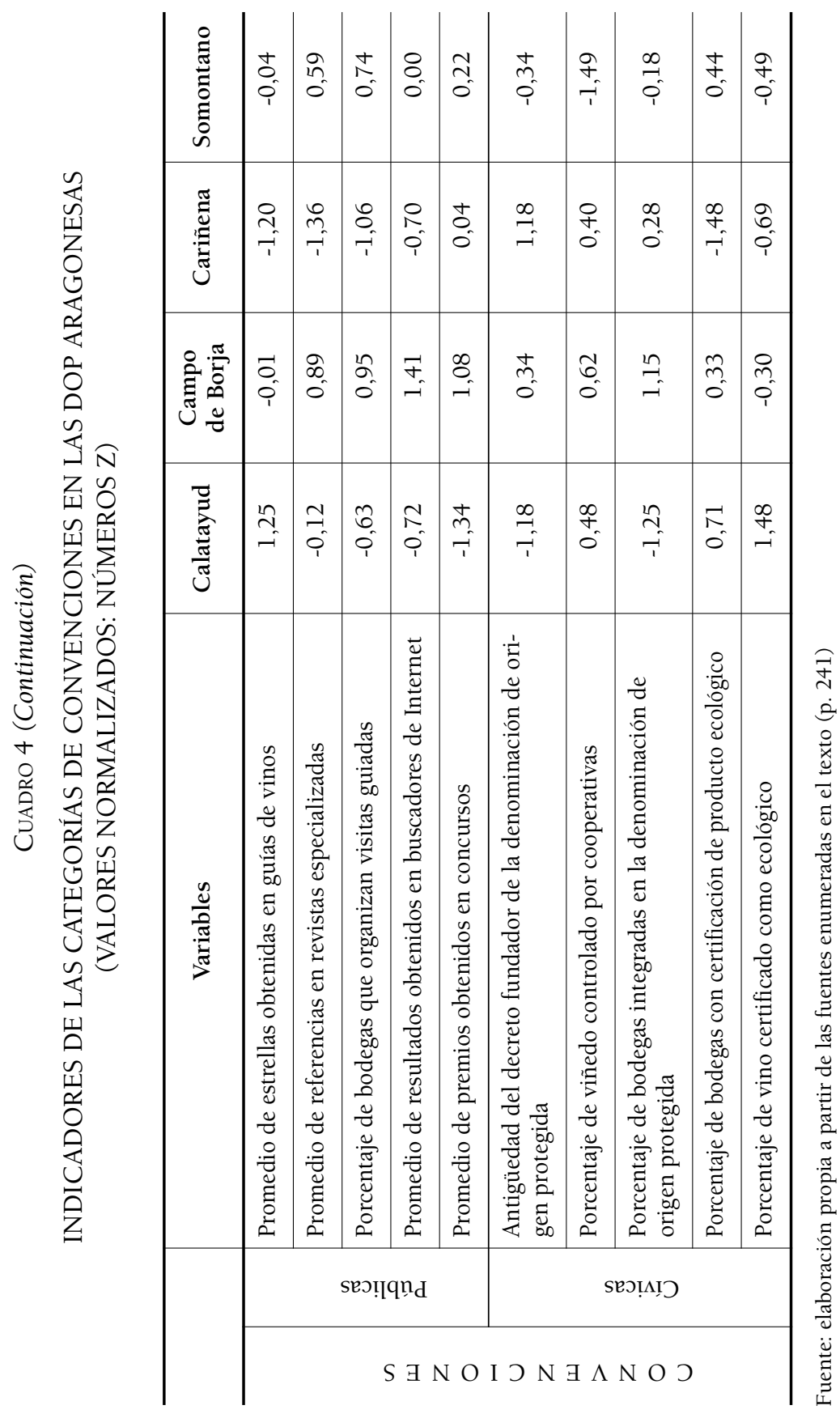

Estudios Geográficos, Vol. LXXIV, 274, pp. 231-254, enero-junio 2013 ISSN: 0014-1496, eISSN: 1988-8546, doi: 10.3989/estgeogr.201308 
FIGURA 2

\section{CONVENCIONES EN LAS DOP ARAGONESAS}

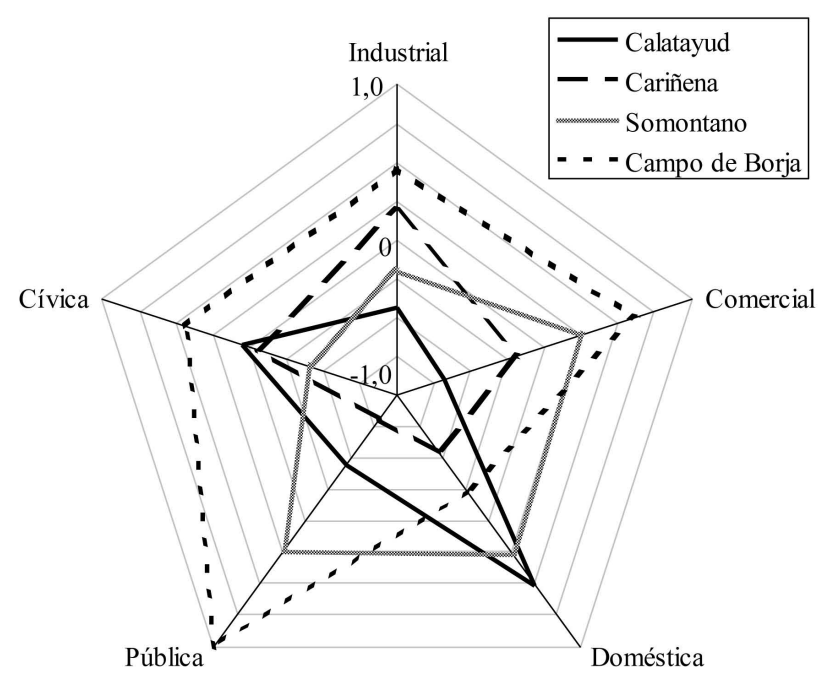

Fuente: elaboración propia a partir de los índices Z que figuran en el cuadro 3.

unas y otras son bastante evidentes: Cariñena presenta valores positivos sólo en las convenciones industriales. Campo de Borja, por el contrario, los presenta en todas, excepto las domésticas. Somontano lo hace en las comerciales, domésticas y públicas, y Calatayud en las domésticas y cívicas. Cabe señalar además que Campo de Borja alcanza los valores más altos de todas las DOP en las categorías de convenciones industriales, comerciales, públicas y cívicas, mientras que en las domésticas los valores más altos son los de Calatayud. Se trata, aparentemente, de cuatro perfiles distintos, que reflejan diferentes estrategias en la búsqueda de la calidad: Cariñena y Calatayud presentan perfiles más simples, frente a Somontano y Campo de Borja, que los tienen más complejos.

La DOP Cariñena entiende la calidad prioritariamente desde las convenciones industriales. Las variables en las que más destaca son la producción media de vino por bodega y el porcentaje de vino joven sobre el total producido; la primera refleja la existencia de bodegas de gran tamaño, en relación con las demás DOP, lo cual facilita la consecución de economías de escala; la segunda refleja que las bodegas tienden a concentrarse en los procesos productivos de mayor simplicidad; en ambos casos, se trata de pautas coherentes con el principio de productividad, que es el concepto clave para las empresas en esta cate- 
goría de convenciones. Otro aspecto en el que destaca es el bajo precio medio del vino, lo cual deriva de las dos variables anteriores. Teniendo en cuenta que esta DOP exportó el 57\% de su producción en la campaña 2008-2009 y que, en cuanto a volumen de exportación, ocupa el primer puesto en Aragón y el sexto en España, puede deducirse que en los mercados internacionales se valoran bien los vinos cuya calidad reposa en convenciones industriales.

La DOP Calatayud, por el contrario, entiende la calidad preferentemente según las convenciones domésticas, en esa nueva alternativa de confiar en lo local como valor seguro en el mercado. Las variables en las que más destaca son los porcentajes de bodegas autóctonas, de marcas que hacen referencia a lo local y de viñedo plantado con variedades autóctonas. Las tres son manifestaciones de una clara voluntad de vinculación al lugar y a la tradición. La DOP presenta también valores destacados en los porcentajes de bodegas con certificación de producto ecológico y de vino certificado como ecológico, variables que hacen referencia a modos de producción respetuosos con el medio ambiente y que se han incluido en la categoría de las convenciones cívicas. Esta orientación compagina bien con la primera, en el sentido de que la confianza en los productos locales y tradicionales se refuerza con el valor de la sostenibilidad. Se trata, por tanto, de una manera de concebir la calidad del vino muy diferente a la anterior, pero que también tiene su sitio en el mercado internacional, al que la DOP Calatayud destinó el 48\% de su producción en la campaña 2008-2009.

La DOP Somontano enfoca su estrategia de calidad de manera multidimensional. En primer lugar, destaca en variables representativas de la categoría de las convenciones comerciales, como el índice de Weaver, que muestra los segmentos de mercado en que está presente, y los porcentajes de viñedo plantado con variedades de uva globales y de bodegas foráneas; las dos primeras reflejan la preocupación por adaptarse a las demandas de los consumidores, dirigiéndose al mayor número posible de nichos de mercado y adoptando variedades de uva de aceptación universal; la última refleja que la DOP es atractiva para empresarios de fuera, porque han detectado en ella la oportunidad de rentabilizar la inversión. Destaca también en algunas variables de las convenciones domésticas, como los porcentajes de marcas que hacen referencia a lo local y de bodegas que venden in situ; esta última es una muestra de la voluntad por recuperar formas de comercialización ligadas al lugar y la tradición. Finalmente, destacan también en variables relacionadas con las convenciones públicas, como los promedios de referencias en revistas especializadas y de premios obtenidos en concursos, lo que refleja la sólida reputación que tiene esta DOP, sobre todo en el mercado nacional, dado que al internacional destina sólo el $21 \%$ de su producción. Somontano es un buen ejemplo de que la conquista de 
la calidad se puede afrontar simultáneamente de varias maneras, aunque puedan parecer contradictorias, como ocurre, por ejemplo, con el uso de nombres locales para marcas de vinos elaborados con uvas no autóctonas.

La DOP Campo de Borja es la que para conseguir vinos de calidad de cara a variados segmentos del mercado, tanto nacional como internacional, aplica el enfoque más multidimensional. Por lo que respecta a las convenciones industriales, supera a Cariñena en las variables citadas en su caso y además presenta un alto porcentaje de cultivo en espaldera, procedimiento técnico que permite mejorar el rendimiento de las vides y mecanizar más eficientemente las tareas agrícolas. Respecto a las convenciones comerciales, supera a Somontano en el porcentaje de bodegas foráneas y el índice de Weaver de segmentos de mercado; además es la que presenta los valores más altos en la variable que combina el porcentaje de vino que se destina a la exportación y el índice de Gibbs-Martin de los países a los que exporta. En cuanto a las convenciones públicas supera ampliamente a la DOP Somontano en las variables citadas en su caso y además destaca en los promedios de resultados obtenidos en los buscadores de Internet y de estrellas que le otorgan las guías de vinos, especialmente la internacional de Parker. Destaca también, finalmente, en el ámbito de las convenciones cívicas, donde los porcentajes del viñedo controlado por cooperativas y de bodegas integradas en la DOP presentan valores elevados, indicando así un alto grado de cohesión social; en las variables relacionadas con la sostenibilidad, en cambio, se ve superada por Calatayud. En resumen, puede decirse que la DOP Campo de Borja ha optado por una estrategia que afronta el problema de la calidad desde casi todos los puntos de vista posibles, lo que la coloca en la mejor posición del conjunto de las DOP aragonesas de cara al mercado internacional; ello se refleja en que es la que mayor penetración, en términos relativos, tiene en el mismo, al que destinó el 68\% de su producción en la campaña 2008-2009.

\section{DISCUSIÓN Y CONCLUSIONES}

El punto de partida de este trabajo es que la teoría de las convenciones ofrece un marco adecuado para comprender mejor los criterios que se usan en la valoración de la calidad de los vinos y en las estrategias que las empresas adoptan para alcanzar esa calidad. Su aportación principal es una propuesta metodológica basada en la selección de una batería de variables estadísticas que se toman como indicadores de las categorías de convenciones. Dicha propuesta ha sido sometida a prueba mediante un caso de estudio centrado en las DOP de Aragón. Los resultados obtenidos muestran que en un espacio geográ- 
fico limitado, como es la comunidad autónoma aragonesa, se dan tantos enfoques de la calidad del vino como DOP existen, a pesar de que las condiciones ecológicas y la tradición cultural presentan un alto grado de homogeneidad. En Cariñena la calidad se basa en criterios preferentemente industriales, mientras que en Calatayud se apuesta por lo doméstico (tradición, apego a lo local) y lo ecológico. Somontano presenta un perfil más complejo, con lo local en primer plano, acompañado por lo comercial y lo público (una buena reputación). Campo de Borja es la más multidimensional de las cuatro, aplicando a la vez criterios industriales, comerciales, de opinión y cívicos. En coherencia con ello el diagrama radial de la DOP Campo de Borja es el que abarca la superficie más extensa (figura 2), lo cual significa que es la que tiene una visión más amplia de la calidad del vino y, en consecuencia, es la que está mejor preparada para competir en un mercado cada vez más globalizado.

Los resultados obtenidos plantean, no obstante, algunas cuestiones que se prestan a discusión: en primer lugar se ha puesto de manifiesto que las estrategias empleadas no son unívocas y que las DOP, aunque se guíen más en su trabajo por una determinada categoría de convenciones, no obvian las demás en sus procesos de producción y comercialización, así que, en todos los casos, las estrategias son multidimensionales.

Lo apuntado anteriormente tiene que ver, en cierto modo, con la escala de análisis. En este trabajo ha sido la Denominación de Origen Protegida, que es, por definición, un conjunto de empresas individualizadas que, a su vez, interpretan a su manera las señales que en torno al concepto de calidad envía el mercado y, en función de ello, eligen sus propias estrategias. Esto significa que el predominio de las convenciones que se ha mostrado aquí para cada una de las DOP aragonesas puede enmascarar su diversidad interna. Así, en la DOP Cariñena el peso de las cooperativas es tan importante que marca el predominio de la convención industrial para el conjunto, aunque en ella existen bodegas particulares que aplican estrategias basadas en otras convenciones ${ }^{2}$.

Del mismo modo la apuesta de la DOP Campo de Borja por la uva autóctona garnacha tinta parecería abocarla a una estrategia predominantemente doméstica y, sin embargo, ésta es la única categoría en la que no presenta valores superiores a las demás DOP. Su éxito en el mercado internacional y la sólida reputación adquirida en los últimos años tienen mucho que ver con el interés con que expertos como Parker han apadrinado los vinos de esta variedad y, por tanto, indica que la convención de opinión ha orientado la estrate-

${ }^{2}$ La contraposición entre las cooperativas, que dominan claramente la producción en Cariñena, y las bodegas particulares, de menor tamaño, está claramente recogida en Loscertales (2009). 
gia de muchas de las bodegas de esta DOP en busca de mercados en los que esa opinión experta se tiene en alta consideración. El caso de esta DOP, pero también el de otras, ilustra muy claramente el hecho de que las diferentes categorías de convenciones son planos diferentes que en ocasiones convergen.

En la misma línea, el análisis realizado permite detectar contradicciones aparentes en las estrategias de calidad aplicadas, como se observa en el caso de la DOP Somontano, en la que las convenciones domésticas son las dominantes, pero los vinos se elaboran a partir de variedades de uva no autóctonas.

Una observación de carácter metodológico es que los números $Z$ son sensibles al número de individuos considerados, de manera que los valores recogidos en los cuadros 3 y 4 variarían si se introdujeran en el cálculo los datos de otras DOP. Lo que en ningún caso variaría es el orden, por lo que conservarían todo su valor las observaciones realizadas sobre la mejor situación de Campo de Borja, en general, y sobre las posiciones relativas de las demás en las diferentes categorías de convenciones.

Finalmente, el método, basado en el tratamiento estadístico de datos cuantitativos, permite un adecuado acercamiento al complejo mundo de la toma de decisiones en las empresas y su éxito en los mercados. No obstante, parece conveniente contrastar esta información con la obtenida mediante trabajo de campo, entrevistas a expertos y encuestas a productores y consumidores, resaltando así la complementariedad de las aproximaciones cualitativas y cuantitativas.

Fecha de recepción: 27/09/2011

Fecha de aceptación: 19/11/2012

BiBLIOGRAFÍA

Amilien, V., Fort, F. y Ferras, N. (2007): "Hyper-real territories and urban markets: changing conventions for local food. Case studies from France and Norway". Anthropology of Food, Special Issue, 2. Disponible en: http://aof.revues.org/document446.html (Fecha de consulta: 08/03/2013).

Boltanski, L. y Thevenot, L. (1991): De la Justification. Les économies de la grandeur. París, Gallimard.

Castelló Puig, A., Frutos Mejías, L. M., Hernández Navarro, M. L., Loscertales Palomar, B. y Ruiz Budría, E. (2010): "Los paisajes vitivinícolas en la economía rural aragonesa", en F. Leco Berrocal (coord.): Territorio, paisaje y patrimonio rural. XV Coloquio de Geografía Rural. Cáceres, Universidad de Extremadura, pp. 49-61.

Diputación General de Aragón (2010): Análisis del sistema Agroalimentario productivo de Aragón. 2. El sector vitivinícola. Zaragoza, Servicio de Planificación y Análisis de la Consejería de Agricultura. 
Escalona Orcao, A. I., Loscertales Palomar, B. y Climent López, E. (2010): "Nuevos retos empresariales en las áreas rurales. Convenciones y estrategias de las industrias del vino y del calzado en la provincia de Zaragoza”, en: Actas del XII Congreso Ibérico de Geografía. Porto, Universidade do Porto (en prensa).

Escalona Orcao, A. I., Loscertales Palomar, B. y Climent López, E. (2011): “Convenciones, prácticas empresariales y pautas para el desarrollo económico y territorial. Las industrias del vino y del calzado en la provincia de Zaragoza". Anales de Geografía de la Universidad Complutense, 31/2, pp. 77-102.

Ilbery, B. (2001): "Relocalización de los sistemas de producción agroalimentaria en la Unión Europea”. Geographicalia, 39, pp. 5-20.

Ilbery, B., Morris, C., Buller, H., Maye, D. y Kneafsey, M. (2005): "Product, process and place: an examination of food marketing and labelling schemes in Europe and North America". European Urban and Regional Studies, 12, pp. 116-132.

Lindkvist, K. B. y Sánchez, J. L. (2008): "Conventions and innovation: a comparison of two localised natural resource-based industries". Regional Studies, 42/3, pp. 343-354.

Loscertales Palomar, B. (2009): La denominación de Origen Cariñena: una apuesta por la singularidad y la calidad en un mundo globalizado. Zaragoza, Diputación Provincial de Zaragoza, Institución Fernando el Católico.

Ministerio de Agricultura, Pesca y Alimentación (2004): Estrategia para el vino en España 2007-2010. Madrid, Ministerio de Agricultura, Pesca y Alimentación.

Murdoch, J. y Miele, M. (1999): "Back to Nature: changing 'worlds of production' in the food sector". Sociologia Ruralis, 39, pp. 465-483.

Murdoch, J., Marsden, T. y Banks, J. (2000): "Quality, nature and embeddedness: some theoretical considerations in the context of the food sector". Economic Geography, 76, pp. 107-125.

Ponte, S. (2009): "Governing through quality: conventions and supply relations in the value chain for South African wine". Sociologia Ruralis, 49/3, pp. 236-257.

Salais, R. y Storper, M. (1992): "The four 'worlds' of contemporary industry". Cambridge Journal of Economics, 16, pp. 169-193.

Sánchez-Hernández, J. L., Aparicio-Amador, J. y Alonso-Santos, J. L. (2010): "The shift between worlds of production as an innovative process in the wine industry in Castile and Leon (Spain)". Geoforum, 41, pp. 469 - 478.

Stræte, E. P. (2004): "Innovation and changing 'worlds of production'. Case-studies from Norwegian dairies". European Urban and Regional Studies, 11, pp. 227-241.

\section{RESUMEN}

La teoría de las convenciones ofrece un marco científico adecuado para analizar los criterios de calidad de consumidores y productores. El sector del vino se orienta hacia la diferenciación por la calidad. El objetivo de la investigación es identificar los criterios de calidad vigentes en el mercado del vino y las estrategias que siguen las empresas para alcanzarlos. 
Dentro del marco de la teoría de las convenciones se ha diseñado un conjunto de variables estadísticas que, una vez normalizadas como números $\mathrm{Z}$, sirven como indicadores de las estrategias de las denominaciones de origen vitivinícolas en su búsqueda de la calidad. Dicho conjunto se ha puesto a prueba mediante un estudio de caso sobre las denominaciones de origen aragonesas y se han identificado modelos diferentes, unos centrados en convenciones concretas (industriales en Cariñena, domésticas en Calatayud) y otros abiertos a distintas categorías de convenciones (Somontano y Campo de Borja).

Palabras Clave: diferenciación del producto; calidad; teoría de las convenciones; sector vitivinícola; Denominación de Origen Protegida.

\begin{abstract}
The convention theory provides a scientific framework appropriate for the analysis of the quality criteria of consumers and producers. The wine industry tends more towards differentiation by means of quality. The aim of this study is to identify the quality standards currently in force within the wine market and the strategies that companies follow to achieve them.

Within the framework of the convention theory we have designed a set of statistical variables that, once normalized as Z-scores, can be used as indicators of the strategies used by wine designations of origin in their search for quality.

This set has been tested through a case study on Aragonese designations of origin and different models have been identified, ones focusing on specific conventions (industrial in Cariñena, domestic in Calatayud) and others open to different categories of convention (Somontano and Campo de Borja).
\end{abstract}

KEY WORDS: product differentiation; quality; convention theory; wine industry; Protected Designation of Origin.

\title{
RÉSUMÉ
}

La théorie des conventions fournit un cadre scientifique approprié pour analyser les critères de qualité des consommateurs et des producteurs. La production du vin est orientée vers la différenciation à travers la qualité. Le but de cette recherche est d'identifier les critères de qualité en vigueur dans le marché du vin et les stratégies suivies par les entreprises pour les atteindre.

Dans le cadre de la théorie des conventions nous avons conçu un ensemble de variables statistiques qui, une fois normalisé sous la forme de scores Z, peuvent servir comme indicateurs de la stratégie des appellations d'origine vinicoles recherchant la qualité.

Cet ensemble a été testé par une étude de cas sur les appellations d'origine aragonaises et des modèles différents ont été identifiés, certains concentrés sur des conventions spécifiques (industrielles à Cariñena, domestiques à Calatayud), d'autres ouverts aux différentes catégories de conventions (Somontano et Campo de Borja).

MotS CLÉs: différenciation du produit; qualité; théorie des conventions; secteur vinicole; Appellation d'Origine Contrôlée. 